\title{
Article
}

\section{How to Test, Analyze, and Reduce Memory Interference Delay in Modern COTS Multicore Systems?}

\author{
Nizar Msadek \\ University of Luxembourg \\ Interdisciplinary Centre for Security, Reliability and Trust (SnT) \\ Esch-sur-Alzette, Luxembourg \\ Email: nizar.msadek@uni.lu
}

\begin{abstract}
In modern Commercial Off-The-Shelf (COTS) multicore systems, cores can produce several simultaneous memory requests. The processing of such requests over the memory controller negatively impacts the interference delay triggered by running parallel tasks on the platform. In this paper, we propose a software-based testing approach for analyzing memory interference delay, when cores are exposed to extensive read/write requests that access in parallel their Cache Coherent Interconnect. The hardware targeted in this work is the well-known LayerScape QorIQ LS2085A, which can be approached as a potential successor to the Freescale QorIQ P4080. The test analysis was conducted based on a bare-metal operating system that we developed to guarantee a deterministic execution environment at all time points. Our testing was accomplished using a set of carefully designed synthetic benchmarks as well as TACLeBench benchmarks.
\end{abstract}

Keywords: Memory Delay; Multicore Systems; Interference Delay; Real-Time Systems; Testing

\section{Introduction}

While the processing of many simultaneous memory requests generally improves the overall memory performance, there remains a challenge to understand precise timing anomalies related to memory in a context where several applications run concurrently. This is because each memory request is likely to be interfered by other requests. Accordingly, analyzing interference delays in modern COTS multicore systems is an important topic in the real-time research community. The targeted hardware in the present study is the well-documented QorIQ LS2085A, a potential successor to Freescale QorIQ P4080. We examine its memory interference delays as well as their impact on the overall system performance. Our study is elaborated based on the publicly available information provided in [1] [2] [3] [4] [5] [6] [7] [8]. Specifically, our contributions are as follows:

(i) To the best of our knowledge, this is the first analysis study examining memory interference delay with respect to the LS2085A platform.

(ii) We provide a comprehensive analysis of the LS2085A platform to know all essential information about the board, including memory hierarchy, I/O interfaces, and cores.

(iii) In order to support the underlying platform, we design a bare metal operating system in which no preemptions and/or other side effects may disturb the measurements at runtime.

(iv) We experimentally analyze the platform using carefully designed synthetic benchmarks as well as TACLeBench benchmarks.

The paper is organized as follows: Section 2 provides background on the QorIQ LS2085A platform. Section 3 outlines the control mechanisms used for efficient synchronization of cores at runtime. Section 4 presents evaluation results. Section 5 discusses related work. Finally, concluding remarks are presented in Section 6 

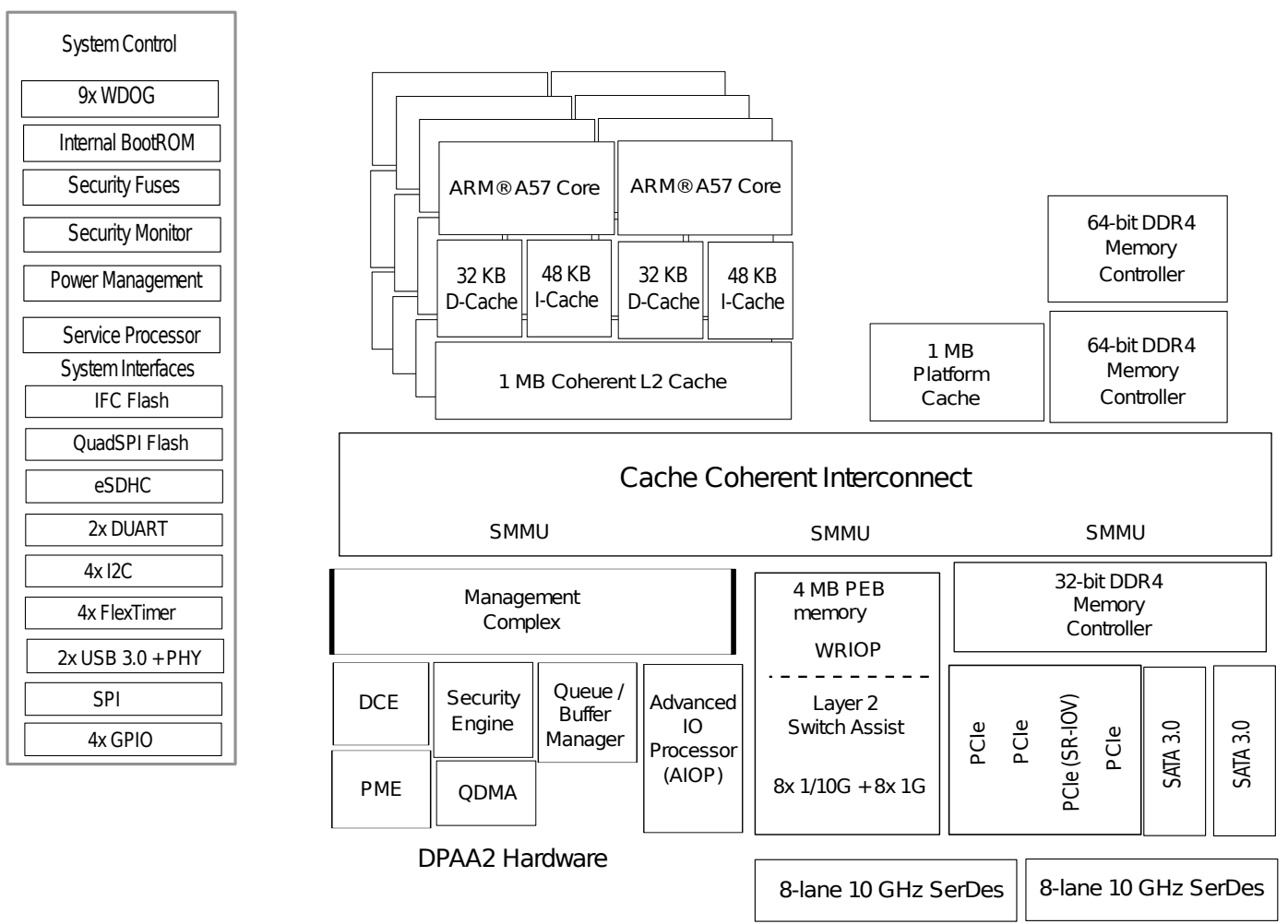

Figure 1. QorIQ LS2085A Board Block diagram (according to [3])

\section{Architectural Description}

Modern COTS-based multicore systems support a high degree of level memory parallelism through a variety of sophisticated components. This section provides the background on these components and reviews existing techniques for achieving shared-memory parallelism. The LayerScape QorIQ LS2085A [1] [2] computing platform is considered a prominent example of modern COTS MPSoC that enables the realization of smarter and predictable safety-critical systems of tomorrow. It is based on ARM technology [9] and belongs to the QorIQ LS2 family of communication processors [10]. It is also a successor to the Freescale QorIQ P4080. Figure1 1 provides an overview of the LayerScape QorIQ LS2085A. Its main parts are further detailed in the below sections.

\subsection{Overview and Memory Hierarchy}

The QorIQ LS2085A board consists of eight Cortex-A57 cores running at $2 \mathrm{GHz}$. Each core has separate L1 data/instruction caches and one unified L2 cache per two cores. The data cache has a capacity of 32 $\mathrm{KB}$, whereas the instruction cache has a capacity of $48 \mathrm{~KB}$. The size of L2 caches is larger than the level one caches, equivalent to $1 \mathrm{MB}$. The cores communicate among themselves and with the main memory over the Cache Coherent Interconnect (CCI). The CCI allows for two memory controllers to connect DDR4 RAM to the cores. Each controller sits behind 1 MB of global L3 cache.

\subsection{Cache Coherent Interconnect}

The Cache Coherent Interconnect (CCI) is responsible for managing and optimizing coherency between caches and the main memory. To the best of our knowledge, only little information is currently available on its implementation protocol. While its implementation remains a black box, investigating the interference delay between cores when these access the same data can prove useful to the purposes of our evaluation. This important aspect helps to ensure non-determinism in the system and consequently to reduce the overestimation of WCETs. 


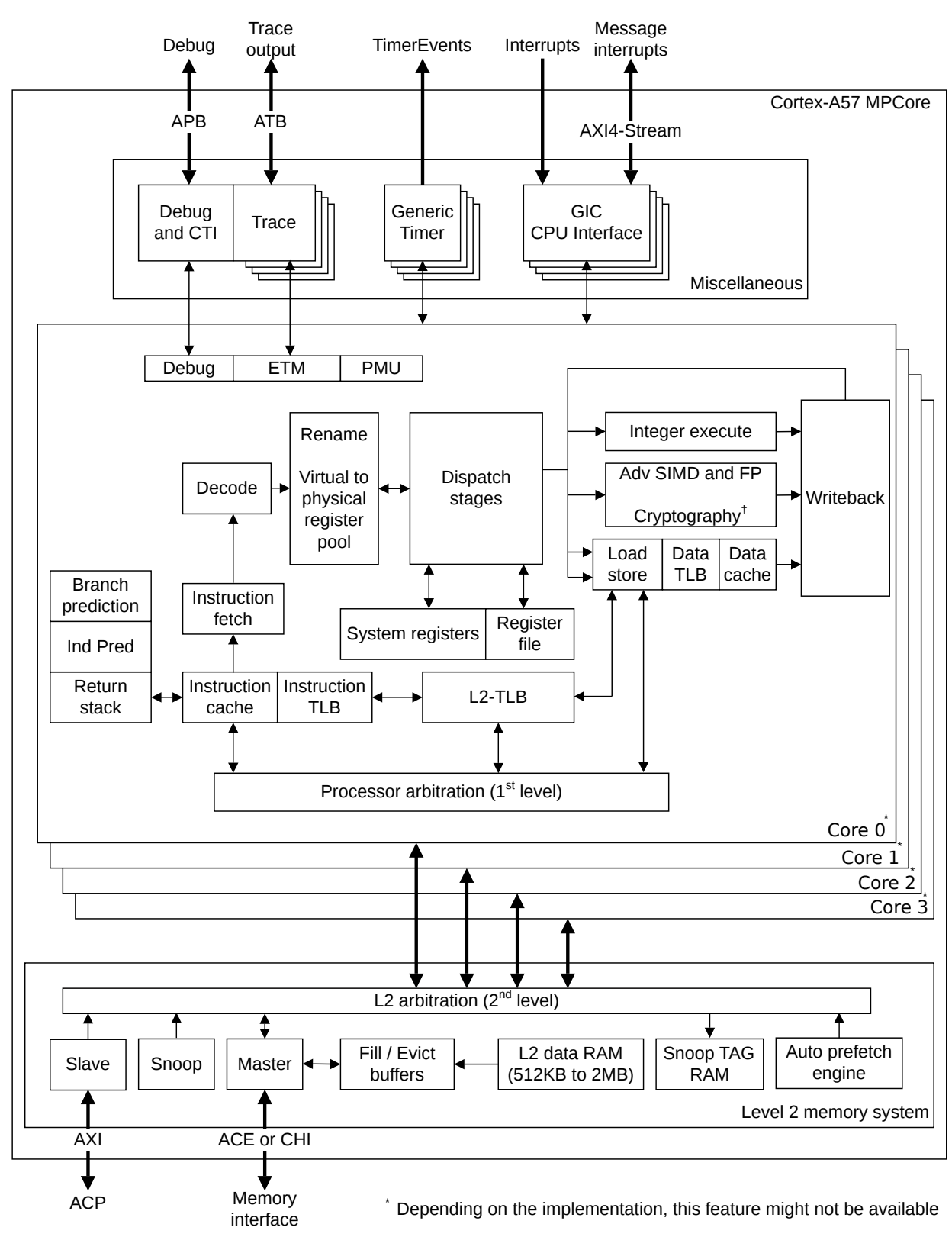

Figure 2. Block diagram of a Cortex-A57 core according to [6]

\subsection{Cortex-A57}

ARM Cortex-A57 [6] is a high-performance, low-power core that belongs to the latest family of A5X-Series. Its implementation is based on ARMv8-A architecture [7] and integrates eight cores in a single multicore device, with L1 and L2 cache subsystems. Figure 2 gives an overview of its main different parts. The QorIQ LS2085A platform considered in this study differs from the implementation depicted in Figure 2 in the number of cores (i.e., eight cores are used instead of four).

\subsubsection{Instruction Fetch}

The Instruction Fetch Engine (IFE) fetches the instructions from the L1 instruction cache and delivers them to the decode engine in up to three instructions per cycle. IFE can support both dynamic and static branch predictions. The L1 instruction cache is a 48KB 3-way set-associative cache characterized by a 64-byte cache line and an optional dual-bit parity protection per 32 bits in the Data RAM as well as 36 bits in the Tag RAM. The IFE also includes a 48-entry fully-associative L1 instruction Translation 
Lookaside Buffer (TLB) that disposes of native support for 4KB, 64KB, and 1MB page sizes. The 2-level dynamic predictor is equipped with a Branch Target Buffer (BTB) for fast target generation.

\subsubsection{Instruction Decode}

The Instruction Decode Engine (IDE) supports both A32 and A64 instruction sets (see Section 3 for further details) as well as the advanced SIMD and Floating-Point instruction sets. It also features a modern technique for register renaming that facilitates out-of-order executions by removing Write-After-Write (WAW) and Write-After-Read (WAR) hazards [11].

\subsubsection{Instruction Dispatch}

The Instruction Dispatch Engine (IDiE) performs checks on the decoded instructions. This is an important task that allows to control in a deterministic way when the pipelines are executed and when the results are retried. The Dispatch Engine supports different types of general purpose registers for both AArch32 and AArch64 states (see Section 3 ) as well as for the advanced SIMD and floating-pint operations.

\subsubsection{Integer Execute}

The Integer Execute Engine (IEE) is responsible for executing all integer type instructions that include:

- Two symmetric Arithmetic Logical Unit (ALU) pipelines

- Integer multiply-accumulate and ALU pipeline

- Iterative integer divide hardware

- Branch and instruction condition codes resolution logic

- Result forwarding and comparator logic

\subsubsection{Load/Store Engine}

The load/Store Engine (LSE) is responsible for managing all load and store operations. It maintains coherency between the L1 and L2 memory systems, and uses a 32KB 2-way set-associative cache with a 64-byte cache line. The latter is characterized by an optional Error Correction Code (ECC) protection per 32-bits. The Load/Store Engine also contains a 32-entry fully-associative L1 data referred as to $\mathrm{TLB}$, which provides support for $4 \mathrm{~KB}, 64 \mathrm{~KB}$, and $1 \mathrm{MB}$ page sizes.

\subsubsection{L2 Memory System}

The L2 memory system maintains L1 instruction and data cache misses from each core. It coordinates requests from the AXI4-Stream, the ACE/CHI master interface and the ACP slave interface. L2 memory features the following capabilities:

- L2 cache with:

- 512KB, $1 \mathrm{MB}$, or $2 \mathrm{MB}$ configurable size

- 16-way set-associative cache with data ECC protection per 64-bits

- Duplicate copy of L1 data cache Tag RAMs from each core for handling snoop requests

- 4-way set-associative of 1024-entry L2 TLB in each core

- Automatic hardware prefetcher with programmable instruction fetch and load/store data prefetch distances

\section{System Control}

The ARM Cortex-A57 core can make use of two processing states, namely the 32-bit and 64-bit processings [12]. The ARMv8 architecture of the 32-bit processing is referred to as AArch32, while the architecture of the 64-bit processing is referred as to AArch64. Since this core can run in two states, it provides capability to execute applications in 64-bit code while also maintaining compatibility with the 


\begin{tabular}{lll}
\hline Register & Width & Description \\
\hline R0-R14 & 32-bit & general-purpose registers \\
SP & 32-bit & stack pointer, alias for R13 \\
LR & 32-bit & link register, alias for R14 \\
PC & 32-bit & exposed program counter \\
S0-S31 & 32-bit & floating-point registers \\
D0-D31 & 64-bit & floating-point/SIMD registers \\
Q0-Q31 & 128-bit & SIMD registers \\
\hline
\end{tabular}

Table 1. AArch32 Registers

\begin{tabular}{lll}
\hline Register & Width & Description \\
\hline & & \\
W0-W30 & 32-bit & general-purpose registers \\
X0- X30 & 64-bit & general-purpose registers \\
LR & 64-bit & link register, alias for X30 \\
SP & 64-bit & stack pointer \\
XZR & 64-bit & zero register \\
V0-V31 & 128-bit & floating-point/SIMD registers \\
\hline
\end{tabular}

Table 2. AArch64 Registers

\begin{tabular}{lll}
\hline Inst. & Aarch32 & Aarch64 \\
\hline I.1 & ADD R1, R2, R3 & ADD W1, W2, W3 \\
I.2 & VCMP.F64 D0, D1 & FCMP D0, D1 \\
& VMRS APSR_nzcv, FPSCR & \\
\hline
\end{tabular}

Table 3. Example of processing instructions in Aarch32 and AArch64 (according to[15]).

existing 32-bit code. This Cortex-A57 core is also equipped with a fully out-of-order execution pipeline which enables to process up to 128 instructions in parallel [11]. Each instruction can be broken into micro operations which are dispatched to multiple arithmetic, branch, floating-point, and load/store execution units. The following section provides an overview of the register allocation as well as the processing instructions of both AArch32/AArch64 states.

\subsection{Register Allocation}

Tables 1 and 2 illustrate the user-visible registers available on AArch32 and AArch64 execution states. The AArch32 execution state provides fifteen 32-bit registers which are referred to as R0-R14 and sixteen 128-bit single-instruction multiple-data registers labeled as Q0-Q15. The SIMD registers are sometimes referred to as NEON registers and can be used in pairs of 64-bit registers from D0 to D31. For instance, D0 and D1 are the lower and higher parts of Q0. It is important to note that all registers delivered by AArch32 are also accessible when the core is operating at AArch64 execution state. Detailed descriptions of the different techniques for mapping registers from AArch32 to AArch64 have been extensively documented elsewhere [13] [14] [4], and do not form part of the scope of this paper. The AArch64 execution state has the additional thirty-one 64-bit registers labeled as X0-X30, and thirty-two 128-bit NEON registers as V0-V31. All registers are accessible at all times, and in all Exception levels. The XZR register is tasked to hold the constant 0 when it is used as a source register, and to discard the result if it is used as a destination register. The Stack Pointer (SP) is mostly used as a load/store base register, and in a small cases for arithmetic instructions to provide access to the current stack pointer. For more details on this topic, please refer to [5].

\subsection{Processing Instructions}

Different processing instructions are available in both AArch32 and AArch64 sates in order to operate values of the general-purpose registers [5] [4]. Most of these instructions are distinguished based on register types. For instance Table 3 , shows the same ADD operand used but with different register types and nevertheless the assembler can automatically choose the correct encoding based on the type of registers and performs the result in R1 and W1, respectively. It is likewise important to note that a one-to-one mapping between instructions in both AArch32 and AArch64 states is not always a straightforward task [15]. This is the case for example when floating point operations are used, as shown in Table 3 . On the AArch32 state, two instructions are needed: one to perform the comparison 
and another to load the result into the condition flag register. The same operation, however, on AArch64 would require only one instruction.

\subsection{Enabling/Disabling Caches}

Some ARMv7-A based cores such as Cortex-A9 require the use of software in order to disable all caches in the system [8]. The use of such software is no longer necessary for the ARMv8-A based Cortex-A57 since the hardware is able to disable automatically the caches after each reset. Nevertheless, in the event of a core powerdown process [8], enabling and disabling caches would prove useful. Therefore, the Cortex-A57 offers a set of operations to interact with caches as shown in Listings 1 and 2.

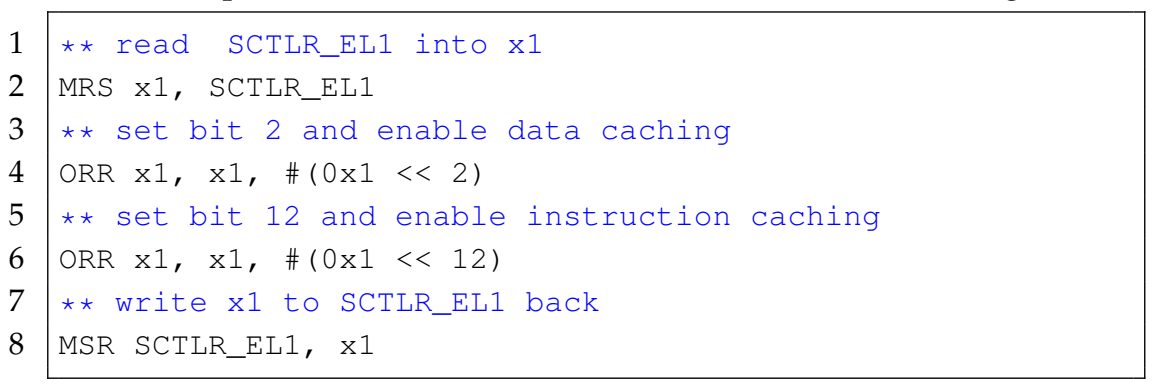

Listing 1: how caches are enabled

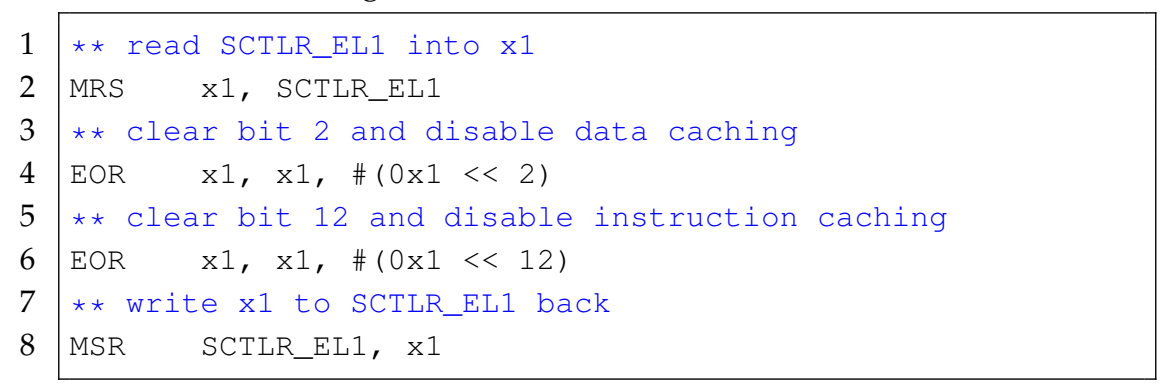

Listing 2: how caches are disabled

The System Control Register (SCTLR) controls the enabling and disabling of caches. If bit 2 and 12 are set, all access permissions are granted and thus the I/D caches are activated. Conversely, if bit 2 and 12 are cleared then caches will be deactivated.

\subsection{I/O interfaces}

LS2085A architecture provides support for different I/O interfaces, which are used to connect with master and slave devices. Master devices are essentially controlled by master interfaces. These interfaces are able to perform memory accesses via the Coherent Interconnect. Examples of such master interfaces include the Peripheral Component Interconnect Express (PCI/PCIe) and the Serial AT Attachment (SATA). Because of their direct link with the Interconnect, master interfaces can potentially impact the interference delays of cores at runtime. The slave interfaces cannot initiate memory access on their own as is the case of master interfaces. They are rather triggered by software. Slave interfaces include Dual asynchronous receiver/transmitter (DUART), Serial Peripheral Interface (SPI), and Inter-Integrated Circuit $\left(\mathrm{I}^{2} \mathrm{C}\right)$.

\subsection{Synchronization Mechanisms}

Synchronization between cores in their access memory is necessary for maximizing utilization at runtime. Cortex-A57 attempts to perform this - among others - by optimizing the order of instruction executions and data accesses, e.g., by optimizing the sequence of instructions as presented in Listings 3

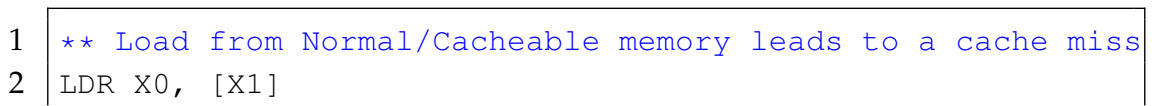




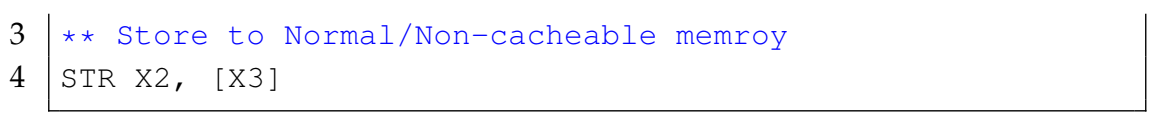

\section{Listing 3: In-Order execution of instructions}

Let us assume that the first instruction we wish to execute leads to a cache miss. As a result, the processor would wait many cycles for the load to complete before executing the store instruction. In Cortex-A57, this delay can be significantly reduced. To do so, the processor recognizes where no dependence exists between the instructions and then executes the store instruction before the load instruction. In some cases, however, these speculative reads or out-of-order executions are not desirable since they can lead to unintended program behavior. For this purpose, synchronization mechanisms are needed. Some of them are introduced as follows:

\subsubsection{Primitive Instructions}

Cortex-A57 core provides primitive instructions to perform atomic memory accesses, including Load and Store exclusive instructions (LDXR/STXR). These are typically used to ensure that multiple processes do not interfere with each other when accessing the same physical address. The LDXR/STXR pair works as follows: LDXR loads a value from a memory address and tries to claim an exclusive lock on this address. When the lock succeeds, the STXR sets afterwards the new value on that location. Both LDXR/STXR instructions are typically used as a basis to implement spinlocks or mutexes (see below the Subsection Mutexes).

\subsubsection{Barriers}

there are different types of barriers supported by the ARM Cortex-A57 core:

- Data Memory Barrier (DMB) forces that all memory accesses before the DMB instruction terminate before any memory access after the DMB instruction starts.

- Data Synchronization Barrier (DSB) terminates when all instructions before the DSB instruction terminate.

- Instruction Synchronization Barrier (ISB) flushes the CPU pipeline in such a way that all instructions coming after the ISB are fetched from cache or memory, once the ISB has been completed.

\subsubsection{Mutexes}

The aforementioned instructions will be made clear using a mutex as an example. A mutex is a flag that enables to access a program block in an atomic fashion. The below listings outlines the implementation of mutex lock/unlock in the Cortex-A57 core using LDXR/STXR.

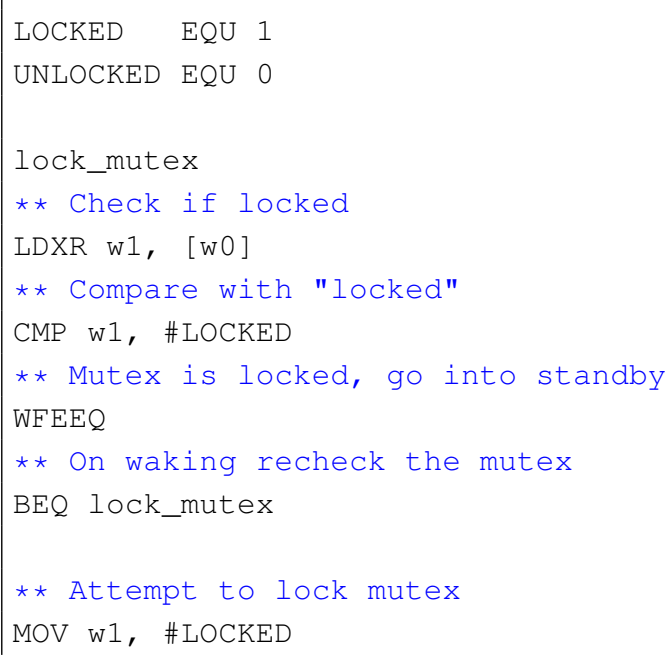




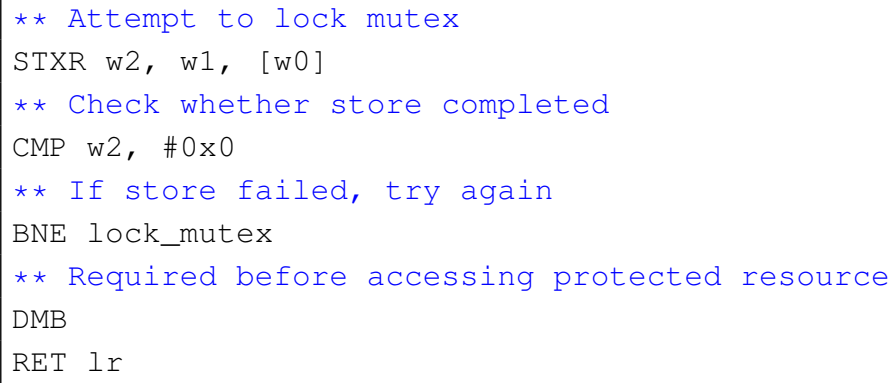

Listing 4: Exemplified implementation of a mutex lock

The lock_mutex function is responsible for acquiring the mutex or blocking until it is acquired. If the mutex is blocked, then all processes must wait for an event WFEEQ before retrying. The function first executes a Load-Exclusive instruction to get the address passed in W0. The value of this address is then compared with locked. If the mutex is locked, the process is momentarily halted before invoking the function lock_mutex again, otherwise, it performs a Store-Exclusive of the value locked. In the case that the Store-Exclusive operation succeeds, the process will execute a DMB and return.

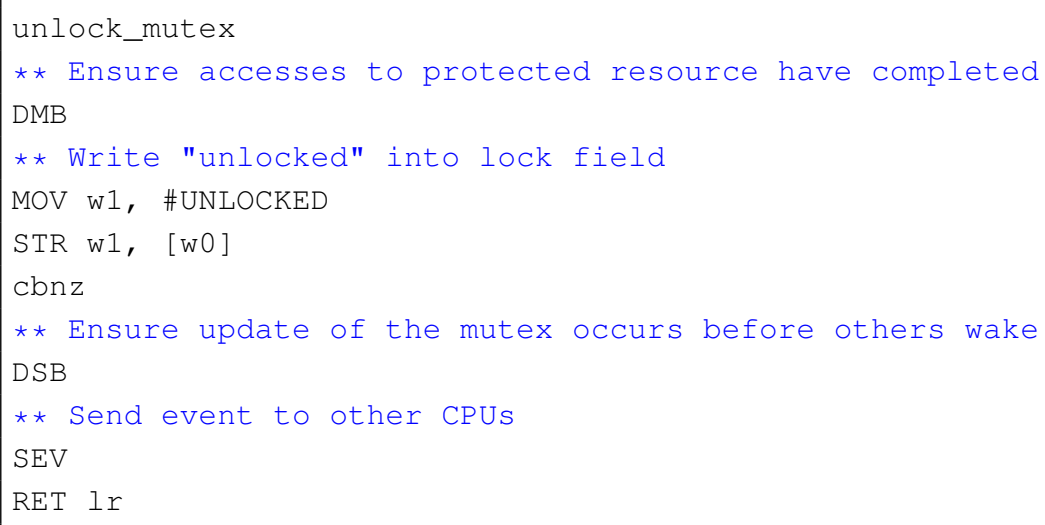

Listing 5: Exemplified implementation of a mutex unlock

The task of unlock_mutex is to release the mutex and send an event of type SEV in order to notify all processes of the change. Afterwards, the function executes a DMB operation to ensure that all memory accesses are done in that point of time. It writes unlocked and stores the value of unlocked in W1. It should be noted that for the store operation a normal STR instruction is used. This is because only one process is currently holding the mutex. In such a case, a DSB is needed to ensure update before other processes wake. Processes are then notified and the program return.

\section{Evaluation}

This section introduces the methodology used to evaluate the target LS2085 platform. A bare-metal operating system was developed in order to guarantee a deterministic execution environment at all time points. This was a necessary step to avoid any side effects that might occur during the evaluation, and which may impact the overall measurement setup. Using this operating system, a series of measurements were conducted. The focus lies basically on determining the interference delays and memory accesses of cores. The methodology for investigating these measurements contains the following two steps:

- Micro-Benchmarking: in the first step an exploring evaluation is used to examine fundamental properties of the LS2085 platform and its limitations in providing these properties.

- Comparative Benchmarking: within a comparative Benchmarking, the performance of the LS2085 platform applied to a suite of benchmarks is measured. The TACLe Benchmarks are used to perform the measurements. 


\subsection{Micro-Benchmarking}

Following a description of the evaluation setup, measurement results are presented and followed by a discussion on the Micro-Benchmarking.

\subsubsection{Evaluation Setup}

The evaluation setup adopted in the present study is similar to that proposed in [16]. A synthetic interference application was created to constantly trigger load/store instructions for all cores. The application runs on a bare-metal operating system in such a way that no preemptions can disturb the measurements during runtime. The body of the application contains a main loop which is executed within one thread per core. Algorithm 1 shows its implementation in pseudocode. At the beginning,

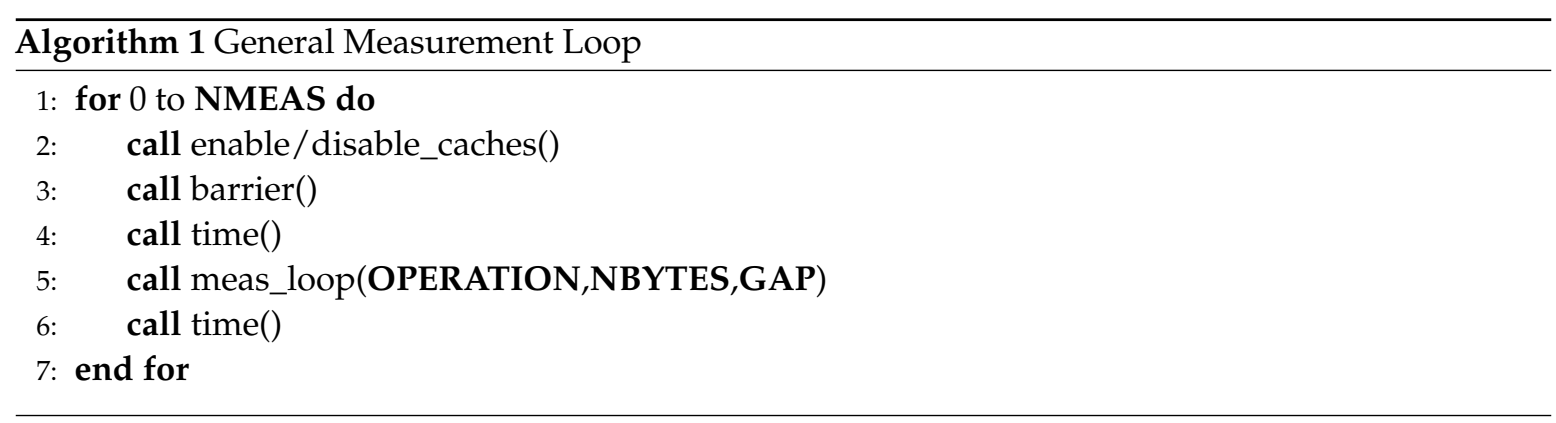

all caches are enabled or disabled depending on the conducted experiment. Then, a barrier is used to start the cores at the same time and thus in parallel. This barrier is implemented according to the concepts presented in Section 3.5. Once the synchronization is complete, the measurement function meas_loop is executed and the time intervals are calculated for each core based on their Time Base Register. The following provides an overview of the parameter settings used:

- NMEAS: stands for the number of replayed scenarios in which the measurements are reported. For this evaluation setup, NMEAS is set to 100 since no significant changes in the results have been observed for larger values of NMEAS.

- Operation: serves to specify the type of operation needed for each conducted experiment. To ensure that the LS2085 platform is evaluated under a great variety of conditions, cores were divided into master and slave cores. For all conducted experiments, only one master is responsible for executing and reporting the results whereas the slaves execute only the routine, and thus in parallel with the master. Since the synthetic application can only issue read or write requests, we decided to consider the following type of operations:

- WM/WS: Write (Master) with concurrent write (Slaves)

- WM/RS: Write (Master) with concurrent read (Slaves)

- RM/WS: Read (Master) with concurrent write (Slaves)

- RM/RS: Read (Master) with concurrent Read (Slaves)

- GAP: is essential to control the cache line size. Obviously, the choice of this parameter is only relevant when the caches are activated. For a cache line size of $64 \mathrm{~B}$, an offset of $64 \mathrm{~B}$ is needed called GAP (see [16] for further details).

In the meas_loop, two macros were used to trigger both of load and store instructions. An exemplary instrumentation of the read macro is shown in Listing 6 . Please note that before calling this macro, the registers $x 0, x 1$, and $x 2$ are initially filled with begin/end address as well as the gap value, respectively.

Listing 6: Macro code for read memory requests

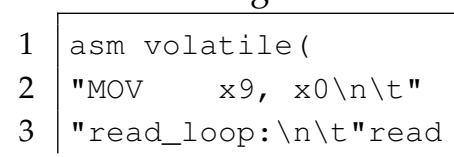




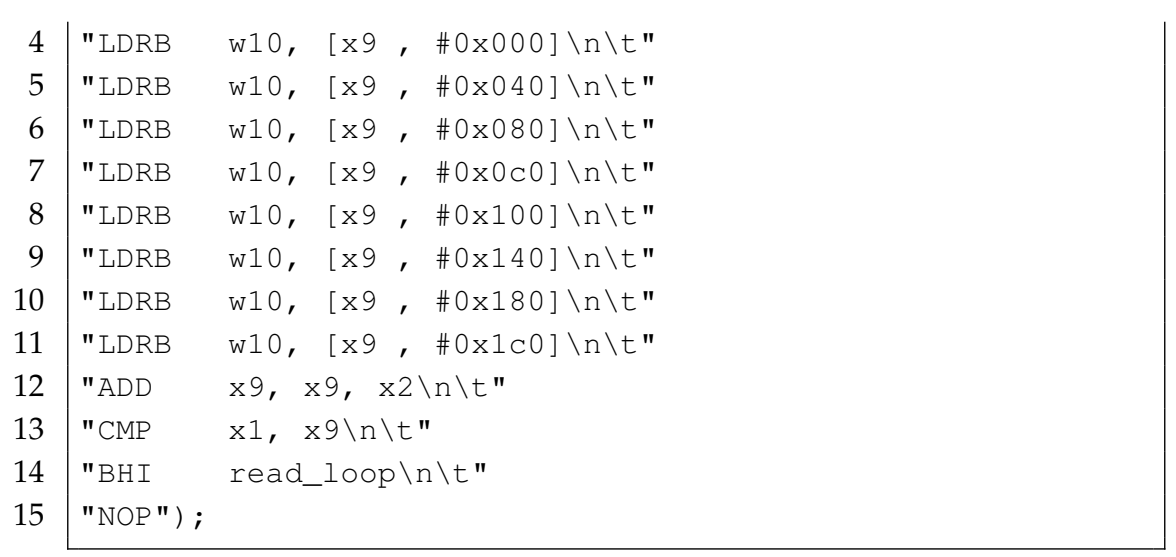

In the first line, the begin address is moved to $\times 9$. Then, a read_loop is called between line 3 and 14 to trigger continuously read accesses in register w10. For each iteration, a gap is added to increment the value of address begin and a comparison is then performed in line 13 to check the end. If the end is reached the macro terminates, otherwise the read_loop is called again. In almost the same manner as the read macro, the write macro has been implemented.

Listing 7: Macro code for write memory requests

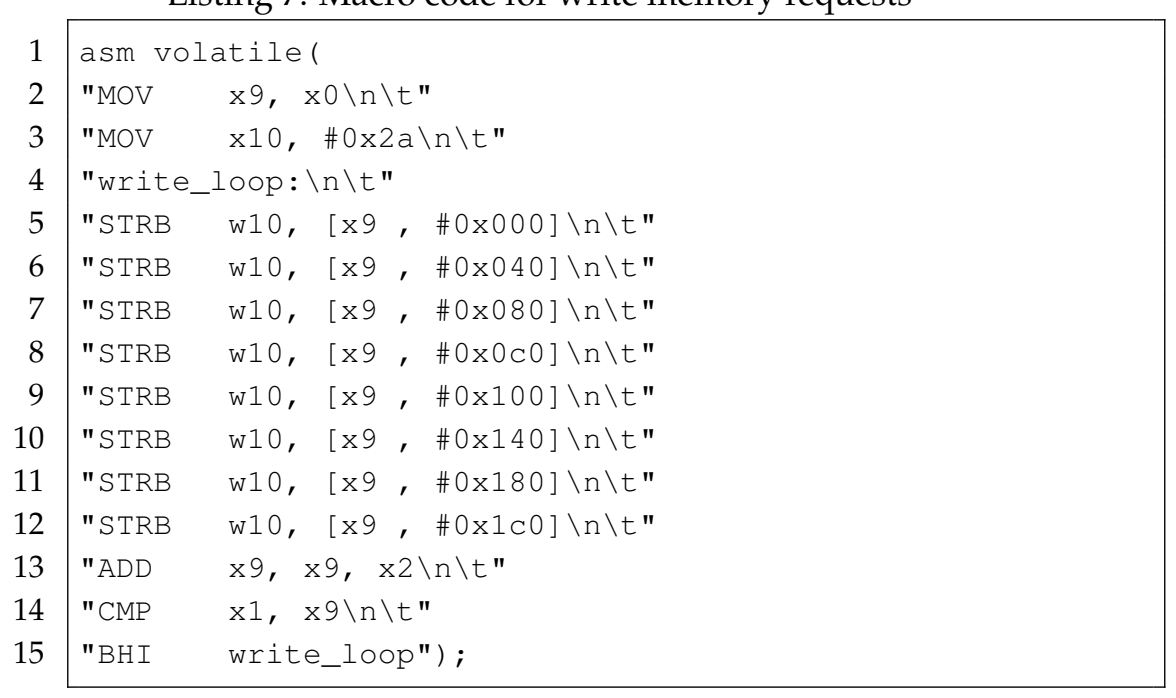

The only difference with the previous example - Listing 6- is that the store instructions are used instead of loads to permanent trigger the write accesses. Listing7/shows an exemplary instrumentation of its code.

\subsubsection{Evaluation Results}

In the following, 8 experiments are presented to examine the performance of the QorIQ LS2085A. The focus lies on interference delays and CPU cycles. It should be noted that interconnect delays and memory access delays cannot be distinguished in the measurements. All experiments were conducted according to the above-mentioned setup, using enabled/disabled caches, and thus for all combinations of read/write operations. To safely upper bound the measured results, the maximum values are used. These values are reported first due to their relevance in estimating the WCETs. Nevertheless, for the sake of completeness, the minimum results have been also added at the end. For all experiments, the values on the horizontal axis stand for the number of active cores. By this means, the term of 1 reflects that only one core is active, 2 that two cores are used, and so on. The term of 8 means that all 8 cores are active. These notations were adopted for all experiments which follow and - depending on the conducted experiment - the performance measurements are always depicted on the y-axis. The first 


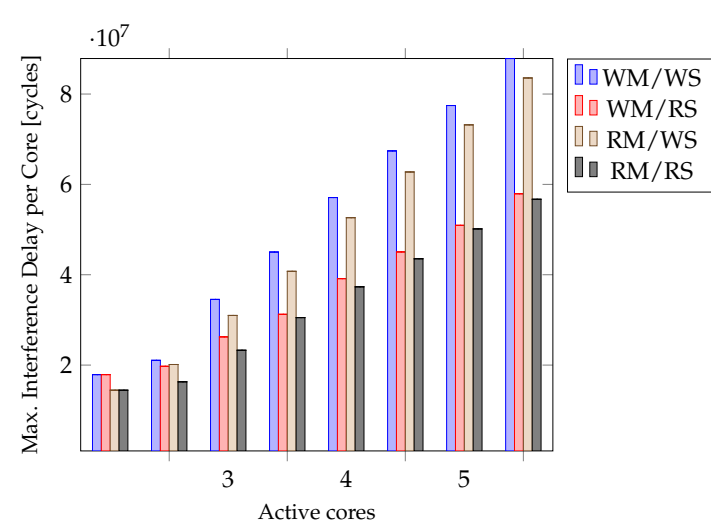

(a) Disabled caches

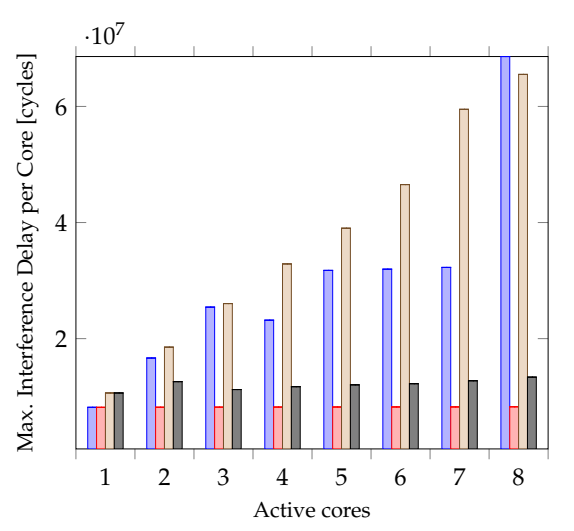

(b) Enabled caches

Figure 3. Maximal interference delay measured with disabled/enabled caches

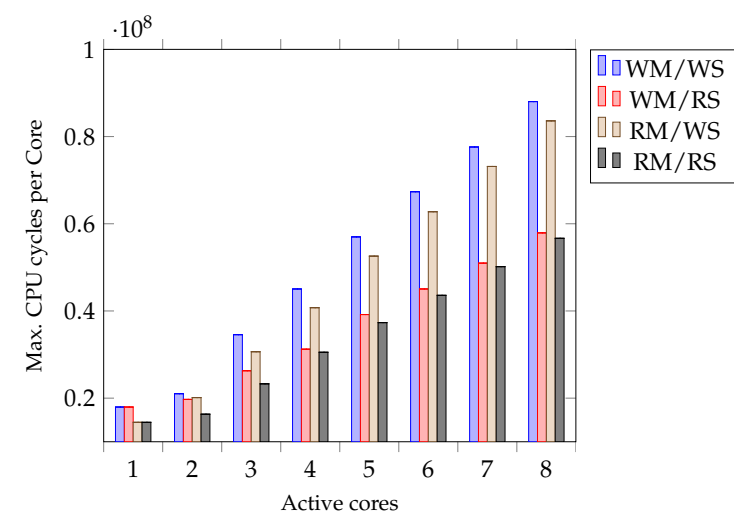

(a) Disabled caches

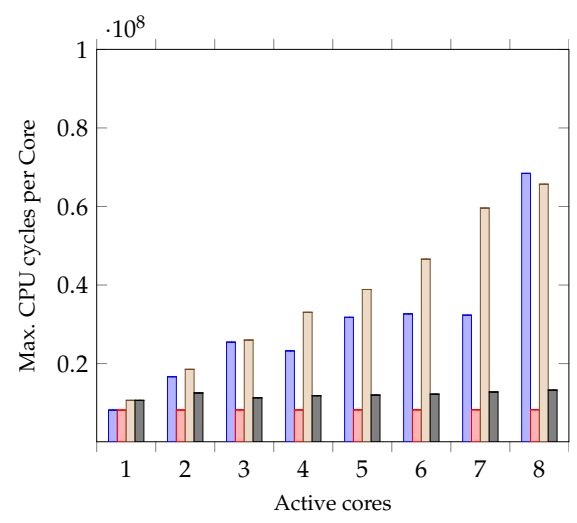

(b) Enabled caches

Figure 4. Maximum number of CPU cycles measured with disabled/enabled caches

four experiments examine the $\max / \mathrm{min}$ performance values evaluated with disabled/enabled caches under the consideration of all Read/Write combinations:

- Experiment 1.a - 1.b: Maximal interference delay measured with disabled/enabled caches (see Figure 3)

- Experiment 2.a - 2.b: Maximum CPU cycles measured with disabled/enabled caches (see Figure (4)

- Experiment 3.a - 3.b: Minimal interference delay measured with disabled/enabled caches (see Figure 5)

- Experiment 4.a - 4.b: Minimum CPU cycles measured with disabled/enabled caches (see Figure6)

\subsubsection{Discussion of the Evaluation Results}

As can be seen from Experiments 1.a - 1.b, the number of active cores plays a major role in influencing the values of interference delays. The greater the number of cores used, the bigger the interference delays. Furthermore, it can be seen from Experiment 1.b that enabling caching indeed generates less overhead delay than with disabled caching. This effect is due to the impact of cache hits which eliminates the need to contact the main memory frequently, and as a consequence, the overhead delay is reduced in the system. Additionally, different values of interference delay were observed for diverse combinations of Read/Write operations. For instance, the WM/WS operations generate more idle time compared to other operations, especially in the case of RM/RS operations. This can be explained by the fact that performing many concurrent write operations would extensively modify data and has as 


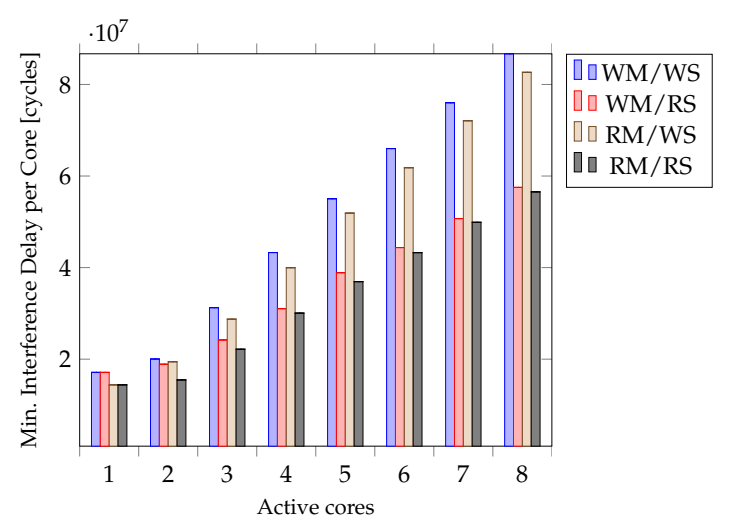

(a) Disabled caches

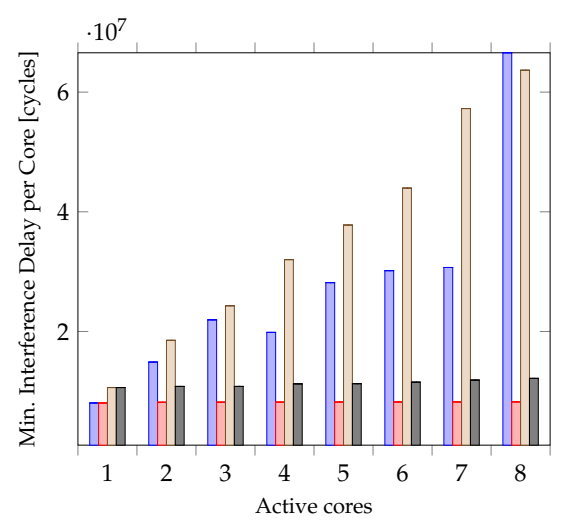

(b) Enabled caches

Figure 5. Minimal interference delay measured with disabled/enabled caches

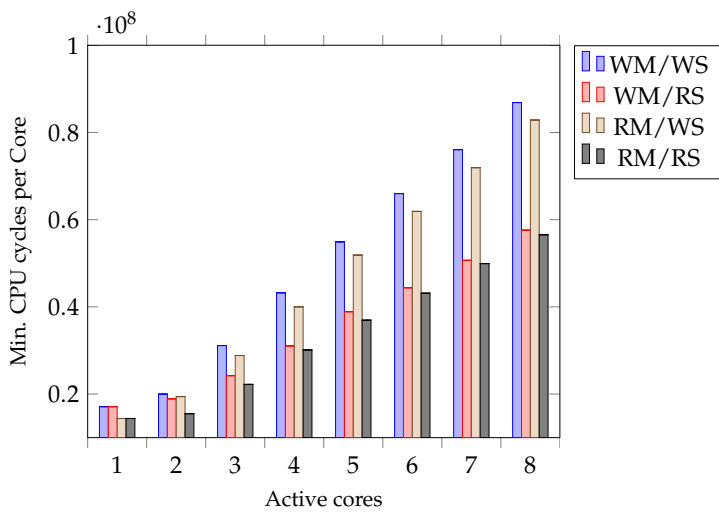

(a) Disabled caches

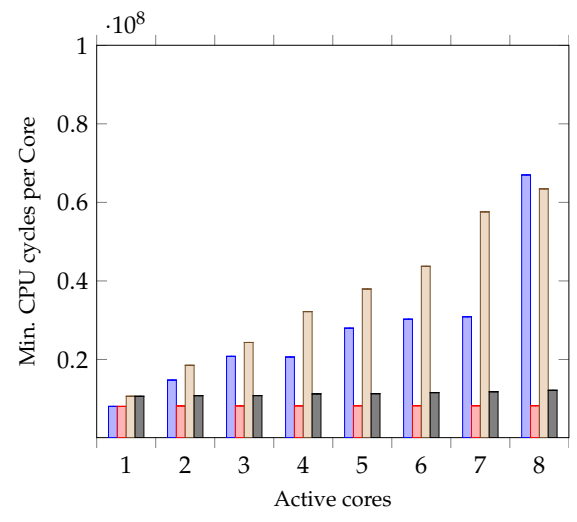

(b) Enabled caches

Figure 6. Minimum number of CPU cycles measured with disabled/enabled caches 
a result to produce also more interference delays and CPU time (see experiment 2.a - 2.b) to achieve coherency.

\subsection{Comparative Benchmarking}

The above introduced setup is now extend by a set of benchmarks to further analyze the performance of the QorIQ LS2085A. This section includes a description of the extended methodology, the experiment results and a discussion.

\subsubsection{Extended Setup}

the aim was to examine the performance of the LS2085A with respect to different benchmarks from the TACLeBench collection [17]. For this purpose, the latest version ${ }^{1}$ of the TACLeBench was used. It consists of a collection of 102 self-contained algorithms - i.e., meaning that no extra dependencies are needed for standard libraries or specific operating systems. This characteristic makes the TACLeBench collection indeed very suitable for our conducted experiments. However, evaluating all the 102 algorithms would go beyond the scope of this paper, we decided therefore to restrict ourselves only to some of them. We selected those algorithms which have a runtime higher than 10000 and less than 1000000 clock cycles. Table 4 gives an overview of the resulted set.

\begin{tabular}{llll}
\hline Name & SLOC & Description & Application Area \\
\hline binarysearch & 47 & Binary search of 15 integers & Controlling \\
fac & 21 & Factorial function & Optimization \\
prime & 41 & Prime number test & Safety/Security \\
bitcount & 164 & Couting number of bits in an integer array & Data Loading \\
fft & 78 & 1024-point FFT, 13 bits per twiddle & Audio/Image Processing \\
insertsort & 35 & Insertion sort & Controlling \\
matrix1 & 28 & Generic matrix multiplication & Optimization \\
md5 & 344 & Message digest algorithm & Safety/Security \\
sha & 382 & NIST secure hash algorithm & Safety/Security \\
\hline
\end{tabular}

Table 4. Benchmark characterization, showing the code size, description and exemplary application areas.

In order to guarantee that all these benchmarks are evaluated under different Read/Write conditions, we decided to consider the following two operations:

- BM/WS: Selected Benchmark (Master) with concurrent write (Slaves)

- BM/RS: Selected Benchmark (Master) with concurrent read (Slaves)

For each operation, one benchmark is selected and executed on a master core (BM) iteratively within a loop of 100 iterations. The synthetic application is solely used to intensify the effect of Read/Write interferences (WS,RS) at runtime. It is executed in parallel on the remaining cores - called slaves until the selected benchmark terminates. And finally, the master takes on the role of reporting the measurements as done before by the micro-benchmarking setup.

\subsubsection{Evaluation Results}

In the following the results of the performance measurements according to the benchmarks that were introduced in Section 4.2.1 are presented. These benchmarks are evaluated within different scenarios of disabled/enabled caches and each of them for both combinations of BM/WS and BM/RS operations. All of the conducted experiments were replayed 100 times and the maximum values are reported first due to their relevance in determining the WCETs. Nevertheless for the sake of completeness, the

1 Version 1.9 - https://github.com/tacle/tacle-bench 
minimum results have also been added at the end. The aspects of interest include the interference delays and cpu cycles. These aspects are always shown on the y-axis depending, on the conducted experiment. Values on the x-axis stand for the selected benchmark with respect to its number of active cores. The representation of four and six cores were omitted for simplicity purposes, since they bring no additional information in interpreting the output results. Therefore, the term of 1 reflects that only one core is active and 8 that all cores are active. The first six experiments examine the maximal interference delays for all benchmarks - evaluated with disabled/enabled caches under the consideration of all Read/Write combinations - whereas the last six experiments consider the performance measurements related to CPU performance.

- Experiment 5.a - 5.c: Maximal interference delay measured with disabled caches. (see Figures 7.,8 and 9

- Experiment 6.a - 6.c: Maximal interference delay measured with enabled caches. (see Figures 10. 11, and 12)

- Experiment 7.a - 7.c: Maximum CPU cycles measured with disabled caches. (see Figures 13, 14 . and 15

- Experiment 8.a - 8.c: Maximum CPU cycles measured with enabled caches. (see Figures 16, 17. and 18)

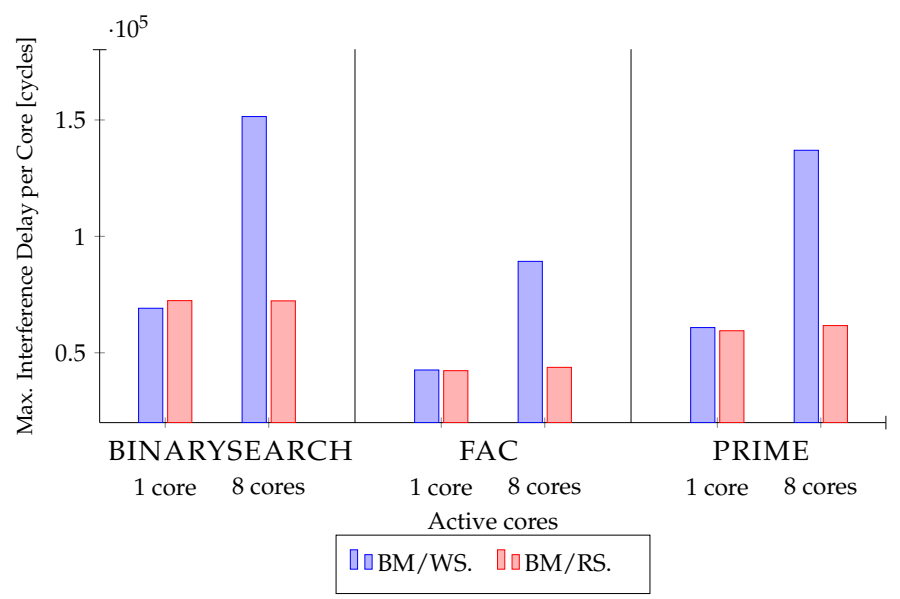

Figure 7. Experiment 5.a: Maximal interference delay measured with disabled caches for one and eight active cores. The considered benchmarks are binarysearch, fac, and prime.

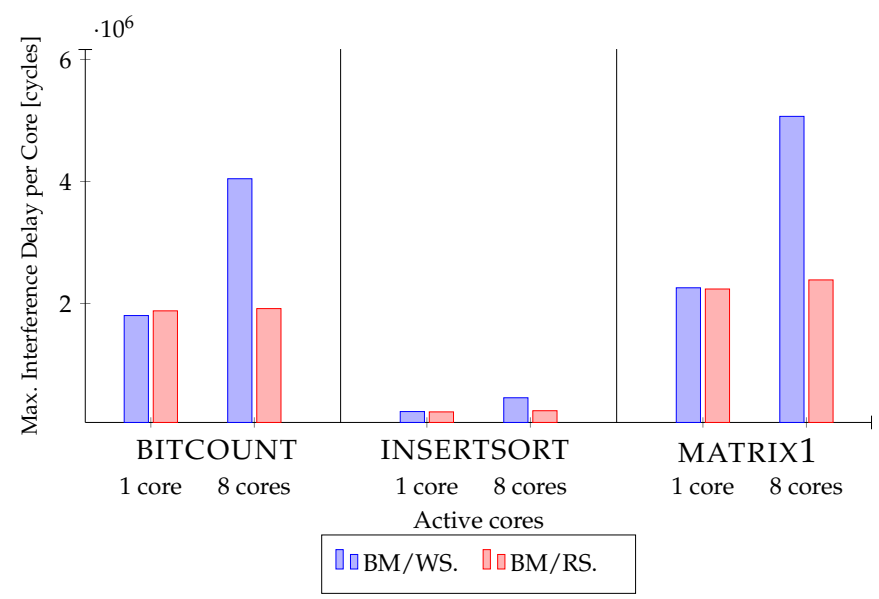

Figure 8. Experiment 5.b: Maximal interference delay measured with disabled caches for one and eight active cores. The considered benchmarks are bitcount, insertsort, and matrix1. 


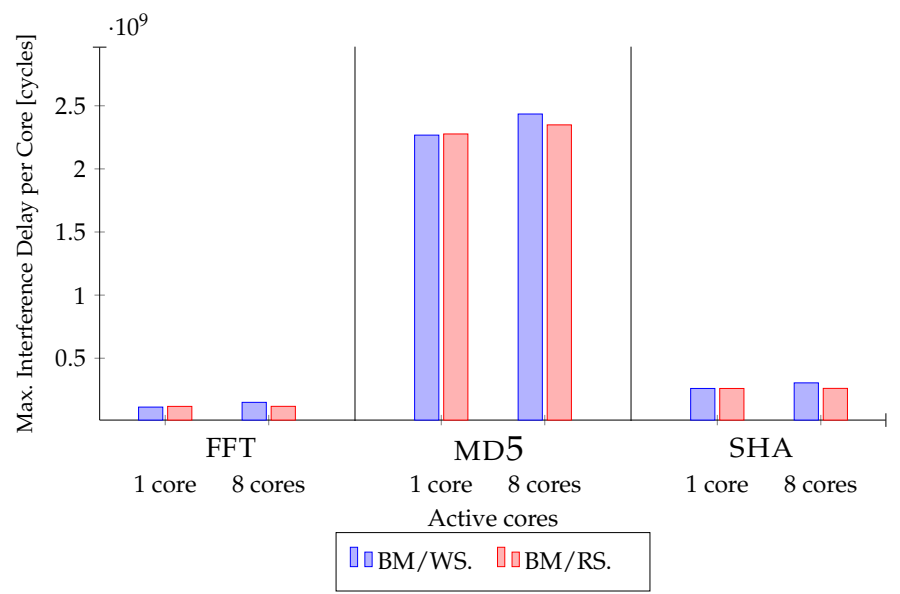

Figure 9. Experiment 5.c: Maximal interference delay measured with disabled caches for one and eight active cores. The considered benchmarks are $\mathrm{fft}, \mathrm{md} 5$, and sha.

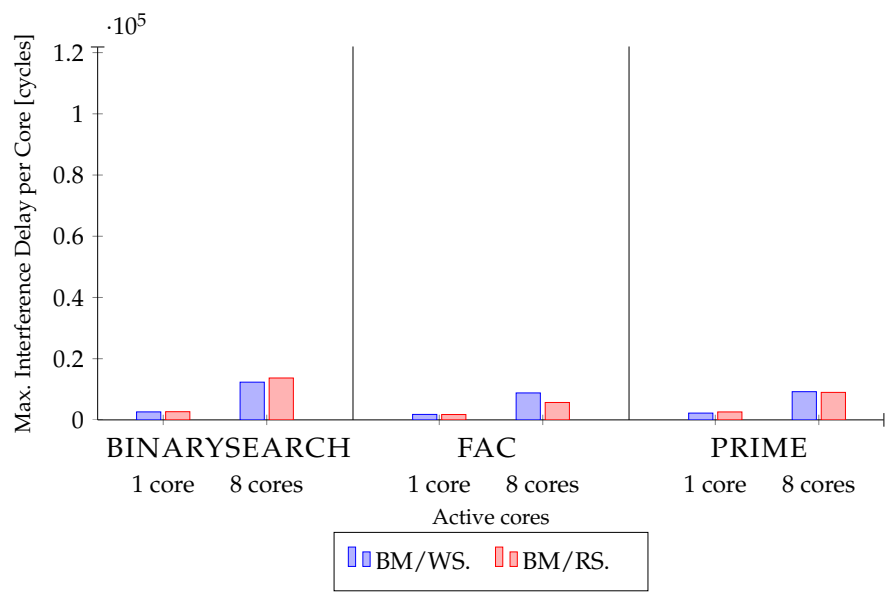

Figure 10. Experiment 6.a: Maximal interference delay measured with enabled caches for one and eight active cores. The considered benchmarks are binarysearch, fac, and prime.

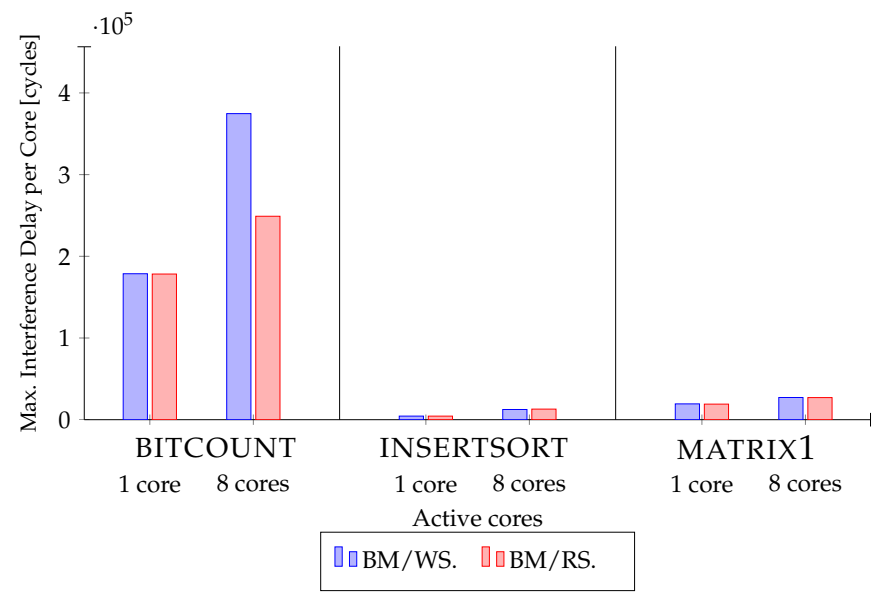

Figure 11. Experiment 6.b: Maximal interference delay measured with enabled caches for one and eight active cores. The considered benchmarks are bitcount, insertsort, and matrix1. 


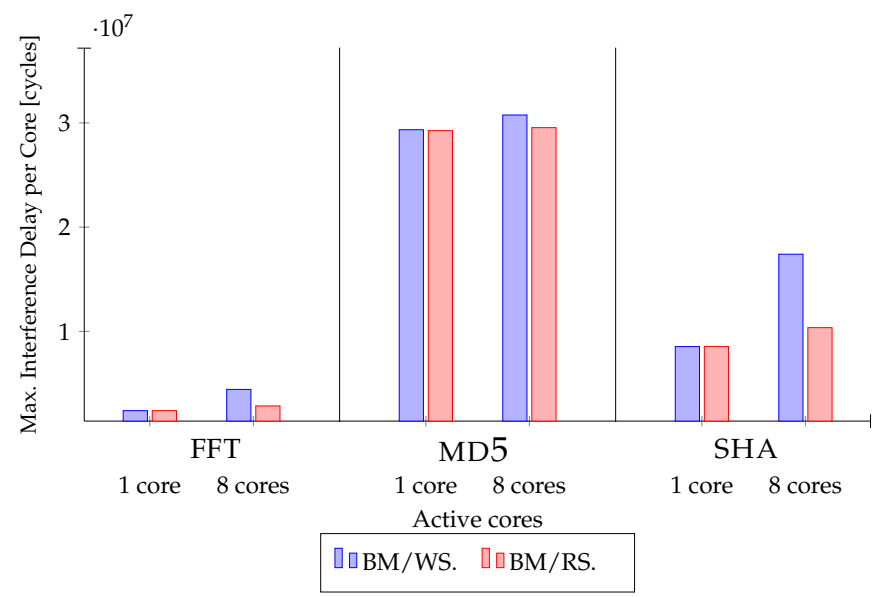

Figure 12. Experiment 6.c: Maximal interference delay measured with enabled caches for one and eight active cores. The considered benchmarks are fft, md5, and sha.

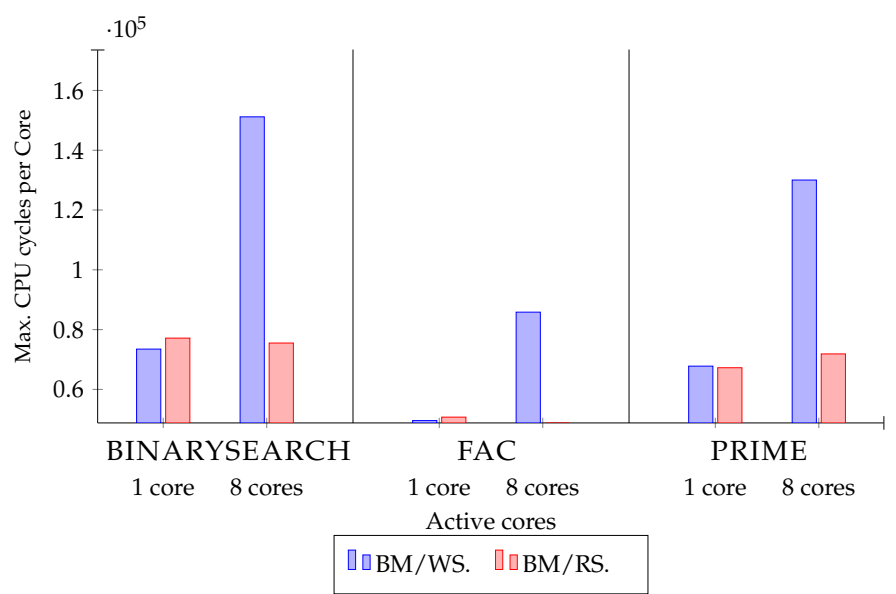

Figure 13. Experiment 7.a: Maximum number of CPU cycles measured with disabled caches for one and eight active cores. The considered benchmarks are binarysearch, fac, and prime.

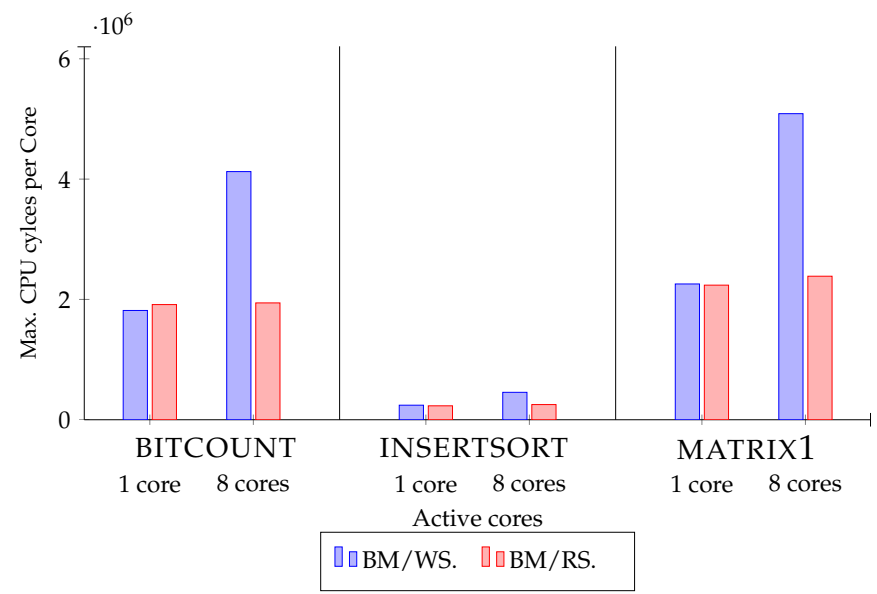

Figure 14. Experiment 15.b: Maximum number of CPU cycles measured with disabled caches for one and eight active cores. The considered benchmarks are bitcount, insertsort, and matrix1. 


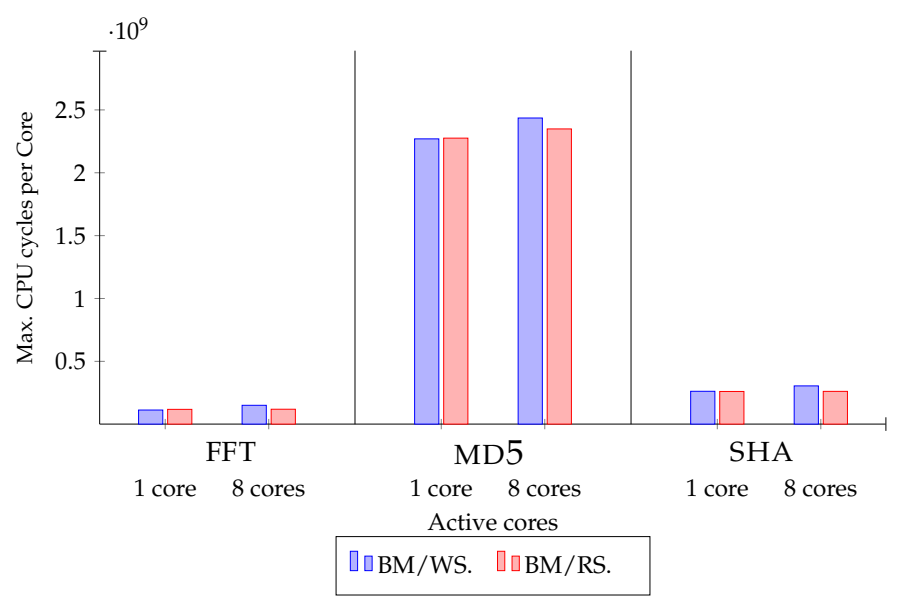

Figure 15. Experiment 15.c: Maximum number of CPU cycles measured with disabled caches for one and eight active cores. The considered benchmarks are fft, md5, and sha.

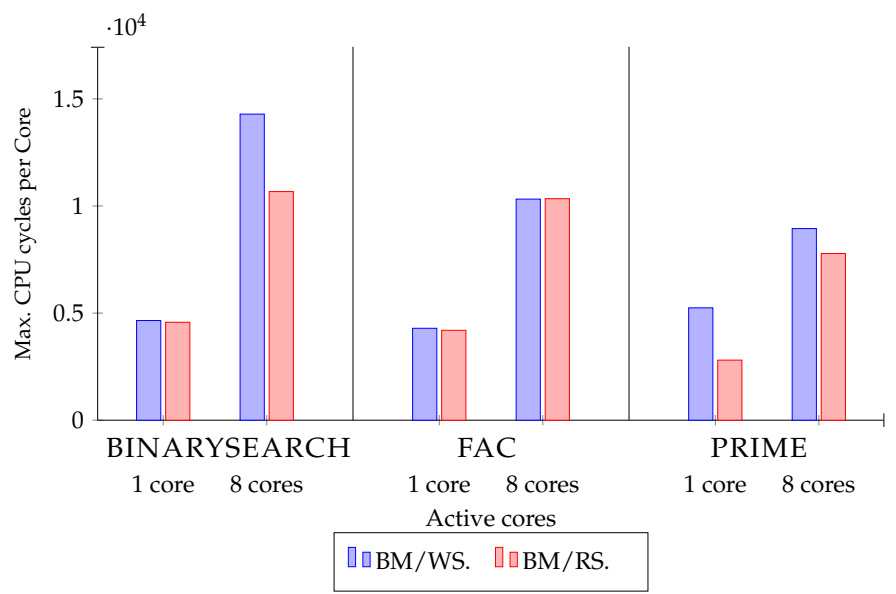

Figure 16. Experiment 8.a: Maximum number of CPU cycles measured with enabled caches for one and eight active cores. The considered benchmarks are binarysearch, fac, and prime.

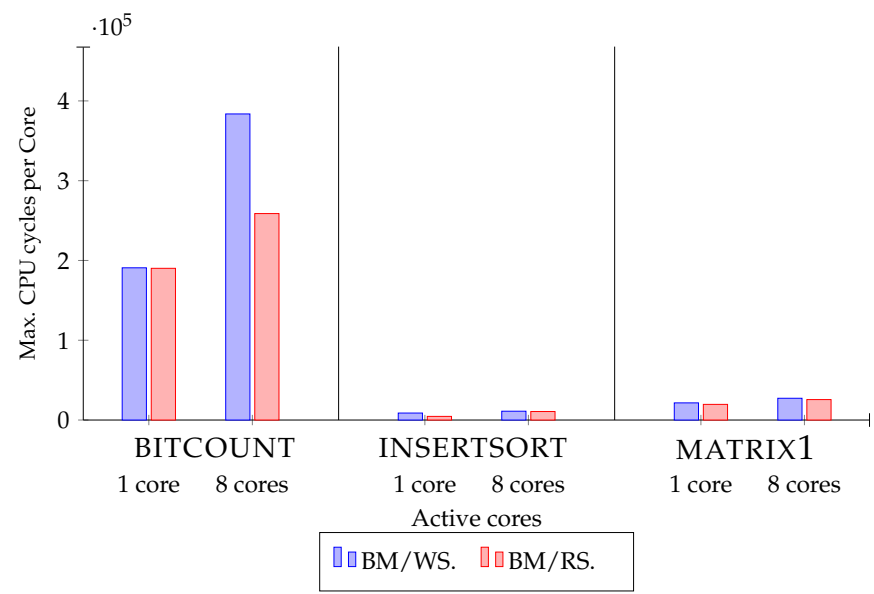

Figure 17. Experiment 8.b: Maximum number of CPU cycles measured with enabled caches for one and eight active cores. The considered benchmarks are bitcount, insertsort, and matrix1. 


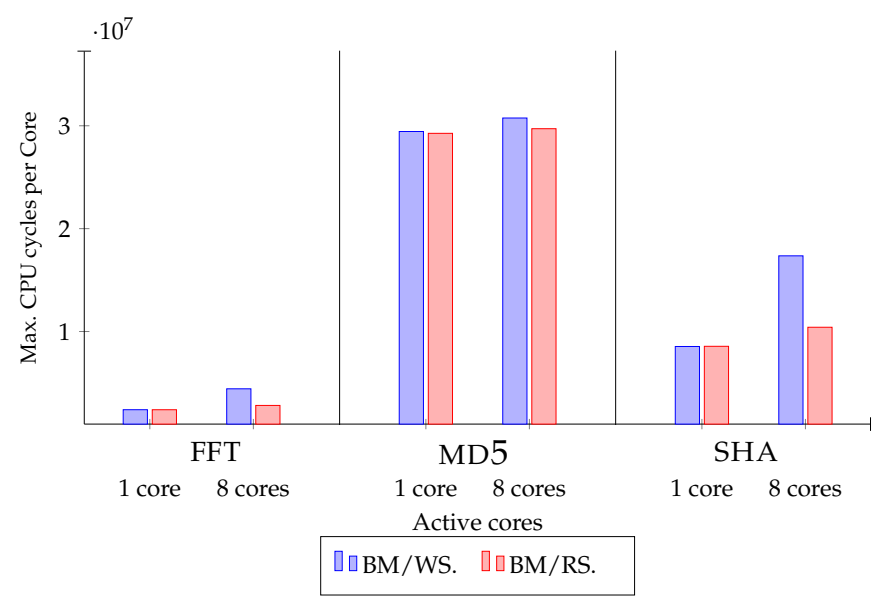

Figure 18. Experiment 8.c: Maximum number of CPU cycles measured with enabled caches for one and eight active cores. The considered benchmarks are $\mathrm{fft}$, md5, and sha.

Now the minimum performance values are presented. The first six experiments examine the minimal interference delays for all benchmarks evaluated with disabled/enabled caches under the consideration of all Read/Write combinations:

- Experiment 9.a - 9.c: Minimal interference delay measured with disabled caches. (see Figures A.1. A.2, and A.3

- Experiment 10.a - 10.c: Minimal interference delay measured with enabled caches. (see Figures A.4. A.5. and A.6.

In Experiments 11.a - 12.c the minimum number of CPU cycles is examined

- Experiment 11.a - 11.c: Minimum CPU cycles with disabled caches. (see Figures A.7, A.8, and A.9

- Experiment 12.a - 12.c: Minimum CPU cycles measured with enabled caches. (see Figures A.10. A.11, and A.12

\subsubsection{Discussion of the Evaluation Results}

As shown in Experiments 5.a - 5.c, the benchmarks differ in how they affect the interference delays. Some are more sensitive than others: MD5 disposes of the highest interference delay, FAC has the lowest one, and the other benchmarks lie in between. This can be seen as evident, since each benchmark has its own characteristic in terms of code structure/size, iteration loop, and input data. Moreover, the number of active cores plays an important role in influencing these delays. The more cores are used, the larger the interferences are. We found as well that the interference delays correlate closely with the used type of Read/Write operations. The WS operations generate in general more slowdown than RS operations. This can be explained by the fact that processing several concurrent write operations would extensively modify data and has as a result to produce also more interference delays and CPU time (see Experiment 7.a - 7.c) to achieve coherency. Furthermore, it can be seen from experiments 6.a 6.c that enabling caching has a positive impact on increasing cache hits, and thus enables to reduce overhead delay in the Cache Interconnect. In Experiments 7.a - 7.c, the impact of this delay reduction on the CPU time was also considered. As expected, the result reveal a good reduction of CPU time, since the coherency was maintained more quickly than with disabled caching and therefore no extra effort is needed from the CPU. Similarly to the maximum delays, the minimum delays in Experiments 9.a - 12.c were investigated and the same conclusion as above was drawn.

\section{Related Work}

As timing performance is becoming increasingly important in modern multicore systems, there have been great interests in the real-time research community to analyze memory related interference delay 
for creating more predictable real-time systems. At the beginning, many computer scientists treat the cost to access memory as a constant. They regard the main memory as a single shared resource by the cores [18] [19] [20] [21]. However, modern COTS-based multicore systems are composed of complex memory components and the memory access cost is far from being a simple constant, since it can change drastically depending on the parameters set by the memory controller to activate components in the system.

The methodology presented in this study differs from state of the art approaches in two fundamental points: 1) we do not consider the main memory as a single shared resource by the cores, and 2) it is not limited to a specific memory component. Moreover, our methodology is software-based, generic, and applicable to any kind of COTS-based multicore Systems. Thus, we want to compare it to approaches that employ similar constraints. The methodology closest to us is [22]. The authors propose an analytical model to examine interference delay in modern multicore systems. Their analysis is based on simulations with assumed non-blocking caches and a DRAM controller that prioritizes reads over writes. However, as these assumptions are not always realistic, they cannot be used to analyze precisely interference delay as we do in this work. Our analysis, in contrast, is conducted on a real COTS multicore platform with a set of carefully designed synthetic benchmarks as well as TACLeBench benchmarks. Moreover, we consider different testing scenarios to generate memory requests at runtime.

\section{Conclusion}

This is the first study on analyzing memory interference delay in the LayerScape QorIQ LS2085A platform. Through micro- and macro-benchmarking, the paper has shown that enabled caching produces less overhead delay than with disabled caching. This is due to the impact of cache hits which eliminates the need to contact the main memory frequently. The paper also experimentally demonstrates that the memory interference delay does not only correlate with the number of used cores, but also with the used type of Read/Write operations. The WS operations generate more slowdown than RS operations. This is explained by the fact that processing several concurrent write operations modifies extensively data and has as a result to produce more interference delays and CPU time. The analysis results in this study can be used in future research designed to estimate more accurately WCETs in industrial multicore scenarios.

\section{References}

1. N. Semiconductors, “Ls2085a/ls2088a reference design board errata,” Board Errata, vol. Rev. 1, no. 27, pp. 1-27, April 2017.

2. N. Semiconductors, "Qoriq ls2085a/ls2088a reference design board getting started guide: Supports ls2085a/ls2088ardb revision f and earlier," Getting Started Guide, vol. Rev. 1, no. 29, pp. 1-29, March 2017.

3. N. Semiconductors, "Design board reference manual," QorIQ LS2085A/LS2088A Reference, vol. Rev. 1, no. 113, pp. 1-113, March 2017.

4. ARM, Programmer's Guide for ARMv8-A, version 1.0 ed. ARM manual, 2015.

5. ARM, ARMv8 Instruction Set Overview, prd03-genc-010197 15.0 ed. ARM Manual, 2014.

6. ARM, ARM Cortex-A57 MPCore Processor, revision: r1p1 ed. http://www.arm.com: Technical Reference Manual, 2013.

7. ARM, ARM Architecture Reference Manual for ARMv8-A, revision: 09-2017 ed. ARMv8-A Reference Manual, 2017.

8. ARM, Bare-metal Boot Code for ARMv8-A Processors, version 1.0 ed. ARM Application Note, 2017.

9. G. Blake, R. G. Dreslinski, and T. Mudge, "A survey of multicore processors," IEEE Signal Processing Magazine, vol. 26, no. 6, pp. 26-37, November 2009.

10. N. Semiconductors, "Qoriq ls2045a and ls2085a," Communications Processors, vol. LS2FAMILYFS REV 4, no. 2, pp. 1-2, 2014. 
11. S. Flur, K. E. Gray, C. Pulte, S. Sarkar, A. Sezgin, L. Maranget, W. Deacon, and P. Sewell, “Modelling the armv8 architecture, operationally: Concurrency and isa," SIGPLAN Not., vol. 51, no. 1, pp. 608-621, Jan. 2016. [Online]. Available: http://doi.acm.org/10.1145/2914770.2837615

12. A. Jalali, R. Azarderakhsh, M. M. Kermani, and D. Jao, “Supersingular isogeny diffie-hellman key exchange on 64-bit arm," IEEE Transactions on Dependable and Secure Computing, vol. PP, no. 99, pp. 1-1, 2017.

13. A. Antras, C. Gorgovan, J. Garside, J. Goodacre, and M. Luján, “Hypermambo-x64: Using virtualization to support high-performance transparent binary translation," SIGPLAN Not., vol. 52, no. 7, pp. 228-241, Apr. 2017. [Online]. Available: http://doi.acm.org/10.1145/3140607.3050756

14. H. Seo, Z. Liu, Y. Nogami, J. Choi, and H. Kim, “Binary field multiplication on armv8," Security and Communication Networks, vol. 9, no. 13, pp. 2051-2058, 2016, sec.1462. [Online]. Available: http://dx.doi.org/ $10.1002 /$ sec. 1462

15. A. Antras, C. Gorgovan, J. Garside, and M. Luján, "Low overhead dynamic binary translation on arm," SIGPLAN Not., vol. 52, no. 6, pp. 333-346, Jun. 2017. [Online]. Available: http://doi.acm.org/10.1145/ 3140587.3062371

16. J. Nowotsch, "Interference-sensitive worst-case execution time analysis for multi-core processors," doctoralthesis, Universität Augsburg, 2014.

17. H. Falk, S. Altmeyer, P. Hellinckx, B. Lisper, W. Puffitsch, C. Rochange, M. Schoeberl, R. B. Sorensen, P. Wägemann, and S. Wegener, "TACLeBench: A Benchmark Collection to Support Worst-Case Execution Time Research," in 16th International Workshop on Worst-Case Execution Time Analysis (WCET 2016), ser. OpenAccess Series in Informatics (OASIcs), M. Schoeberl, Ed., vol. 55. Dagstuhl, Germany: Schloss Dagstuhl-Leibniz-Zentrum fuer Informatik, 2016, pp. 2:1-2:10. [Online]. Available: http://drops.dagstuhl. de/opus/volltexte/2016/6895

18. Z. P. Wu, Y. Krish, and R. Pellizzoni, "Worst case analysis of dram latency in multi-requestor systems," in 2013 IEEE 34th Real-Time Systems Symposium, Dec 2013, pp. 372-383.

19. S. Rixner, W. J. Dally, U. J. Kapasi, P. Mattson, and J. D. Owens, "Memory access scheduling," SIGARCH Comput. Archit. News, vol. 28, no. 2, pp. 128-138, May 2000. [Online]. Available: http://doi.acm.org/10. $1145 / 342001.339668$

20. A. Schranzhofer, J. Chen, and L. Thiele, "Timing analysis for tdma arbitration in resource sharing systems," in 2010 16th IEEE Real-Time and Embedded Technology and Applications Symposium, April 2010, pp. 215-224.

21. G. Yao, R. Pellizzoni, S. Bak, E. Betti, and M. Caccamo, "Memory-centric scheduling for multicore hard real-time systems," Real-Time Systems, vol. 48, no. 6, pp. 681-715, Nov 2012. [Online]. Available: https: //doi.org/10.1007/s11241-012-9158-9

22. H. Yun, R. Pellizzon, and P. K. Valsan, "Parallelism-aware memory interference delay analysis for cots multicore systems," in 2015 27th Euromicro Conference on Real-Time Systems, July 2015, pp. 184-195.

\section{Appendix Further Results}

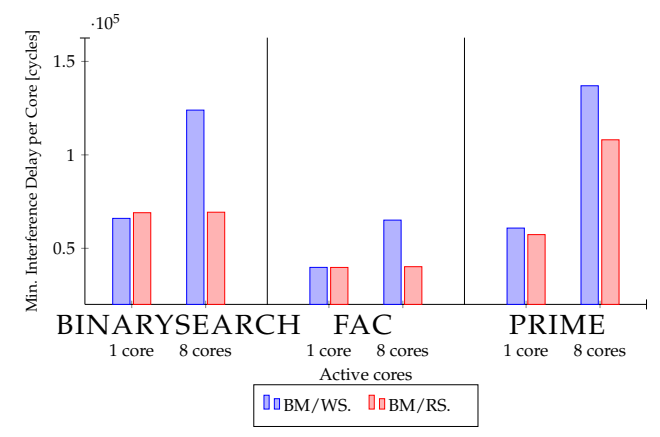

Figure A.1. Experiment 9.a: Minimal interference delay measured with disabled caches for one and eight active cores. The considered benchmarks are binarysearch, fac, and prime. 


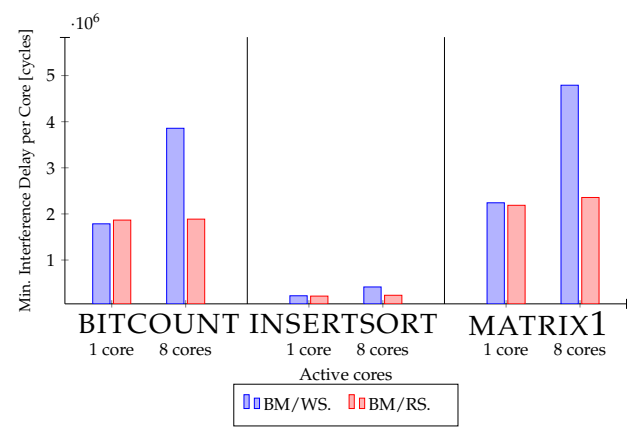

Figure A.2. Experiment 9.b: Minimal interference delay measured with disabled caches for one and eight active cores. The considered benchmarks are bitcount, insertsort, and matrix1.

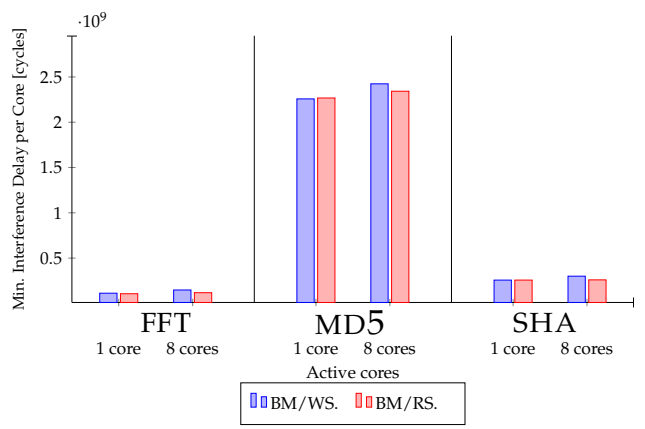

Figure A.3. Experiment 9.c: Minimal interference delay measured with disabled caches for one and eight active cores. The considered benchmarks are $\mathrm{fft}, \mathrm{md} 5$, and sha.

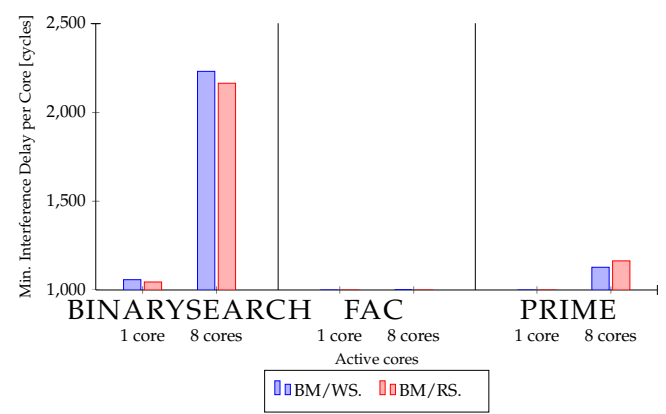

Figure A.4. Experiment 10.a: Minimal interference delay measured with enabled caches for one and eight active cores. The considered benchmarks are binarysearch, fac, and prime.

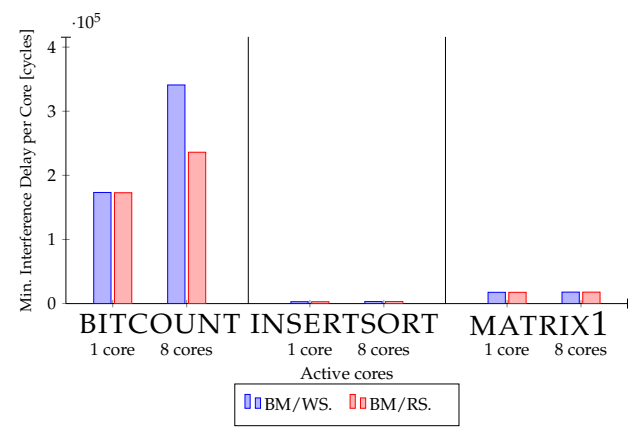

Figure A.5. Experiment 10.b: Minimal interference delay measured with enabled caches for one and eight active cores. The considered benchmarks are bitcount, insertsort, and matrix1. 


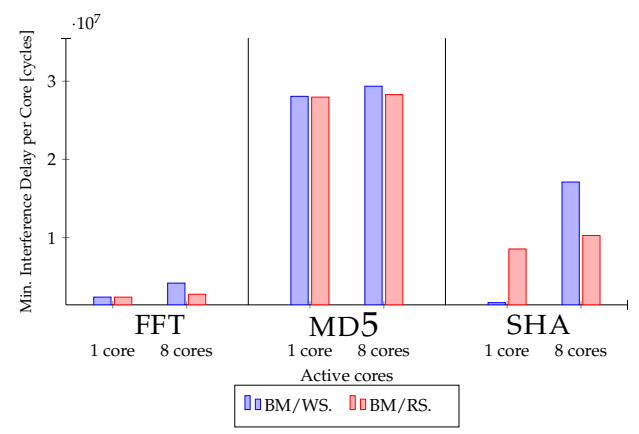

Figure A.6. Experiment 10.c: Minimal interference delay measured with enabled caches for one and eight active cores. The considered benchmarks are $\mathrm{fft}, \mathrm{md} 5$, and sha.

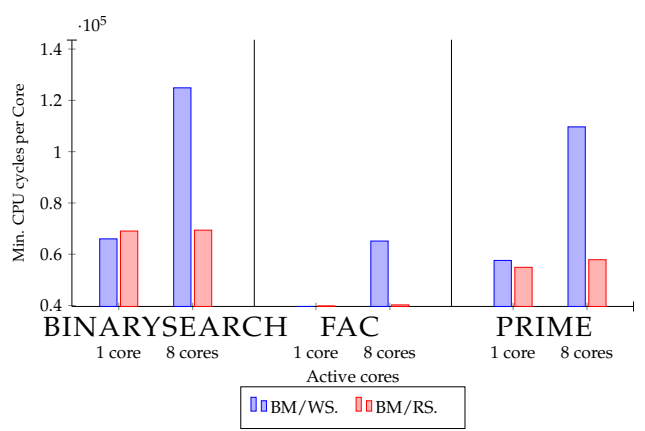

Figure A.7. Experiment 11.a: Minimum number of CPU cycles measured with disabled caches for one and eight active cores. The considered benchmarks are binarysearch, fac, and prime.

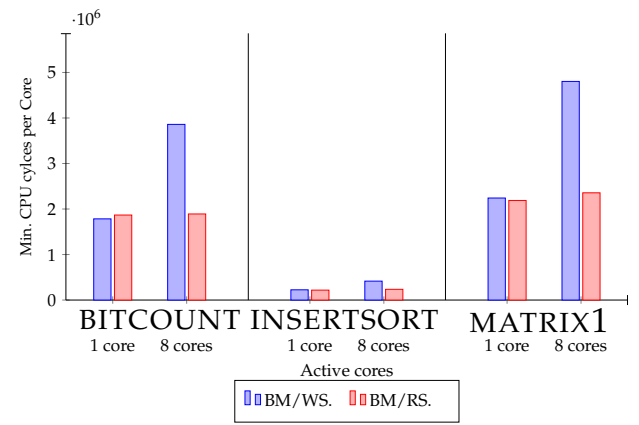

Figure A.8. Experiment 11.b: Minimum number of CPU cycles measured with disabled caches for one and eight active cores. The considered benchmarks are bitcount, insertsort, and matrix1.

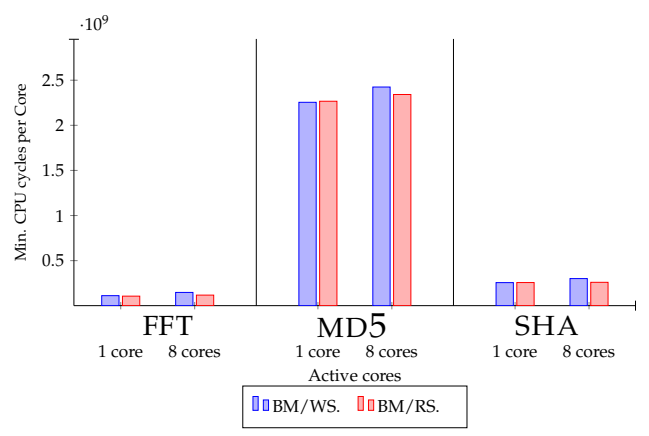

Figure A.9. Experiment 11.c: Minimum number of CPU cycles measured with disabled caches for one and eight active cores. The considered benchmarks are fft, md5, and sha. 


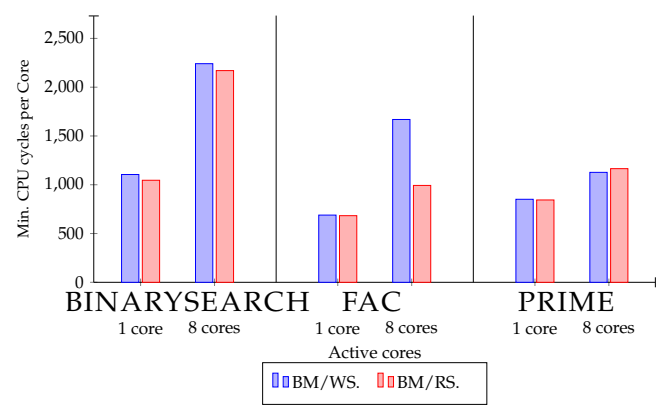

Figure A.10. Experiment 12.a: Minimum number of CPU cycles measured with enabled caches for one and eight active cores. The considered benchmarks are binarysearch, fac, and prime.

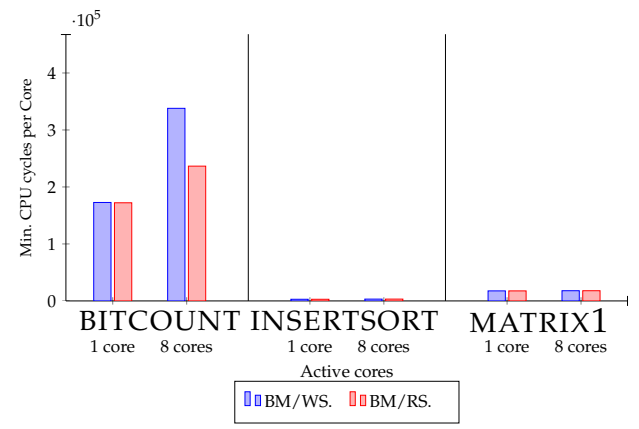

Figure A.11. Experiment 12.b: Minimum number of CPU cycles measured with enabled caches for one and eight active cores. The considered benchmarks are bitcount, insertsort, and matrix1.

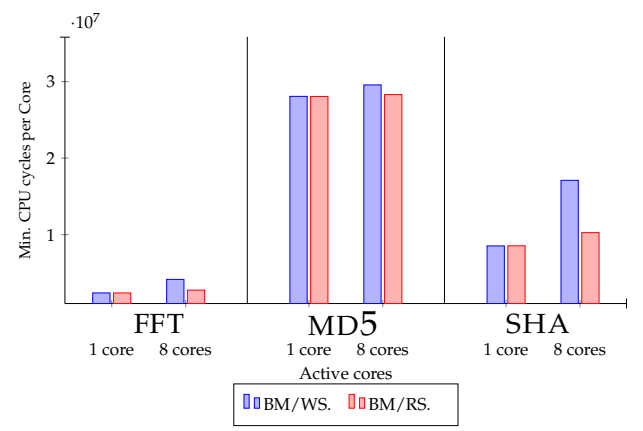

Figure A.12. Experiment 12.c: Minimum number of CPU cycles measured with enabled caches for one and eight active cores. The considered benchmarks are fft, md5, and sha. 